\title{
Improved region growing algorithm for the calibration of flaking deterioration in ancient temple murals
}

\author{
Jianfang Cao ${ }^{1,2^{*}} \mathbb{0}$, Yanfei Li ${ }^{1}$, Hongyan Cui ${ }^{1}$ and Qi Zhang ${ }^{1}$
}

\begin{abstract}
Ancient murals are vulnerable to varying degrees of damage due to long-term exposure to external environmental factors such as light, temperature and humidity. Enabling people to appreciate the original features of murals has become a concern for field experts. The development of computer technology makes it possible to use intelligent information processing to simulate and restore ancient murals. This paper proposes an improved region growing algorithm based on threshold segmentation to automatically calibrate the flaking-related deterioration of murals in response to erosion by taking temple murals from the Song Dynasty in Kaihua Temple as the study object. First, we analyze the color characteristics of the flaking area, mark the suspected flaking-damaged points by threshold segmentation, and use these points as seeds for the area growth and expansion of the flaking area. We then calculate the color mask. Next, in the YCbCr and HSV color spaces, the brightness, chroma, and saturation characteristics of the flaking area are analyzed. The masks for the brightness, chroma, and saturation of the flaking area are obtained by threshold segmentation, and all the feature masks are merged. Finally, the mask of the flaking area obtained by data fusion is added to the original image to calibrate the flaking deterioration. Compared with current calibration algorithms based on multiscale mural deterioration, the experimental results show that the average error and error standard deviation of the proposed calibration algorithm are 1.91 and 1.82, respectively, without noise and 1.97 and 1.85 , respectively, with noise. The errors are reduced, and the calibration performance is improved and stable. This work provides a good foundation for the virtual and practical restoration of ancient murals.
\end{abstract}

Keywords: Flaking deterioration, Threshold segmentation, Region growing, Feature mask, Automatic calibration

\section{Introduction}

Ancient mural art represents an important cultural heritage and includes a substantial amount of cultural, historical, and artistic information that vividly documents the social features of various ethnic groups and periods and has important historical, scientific, and artistic values. Palace relief frescos from the Egypt and Two Rivers Empire ages, palace murals from the Aegean culture, temples, tombs and house murals from Ancient Greece and Rome, and tomb murals, stone murals, and temple murals from the Chinese Han Dynasty, Wei Jin Southern

\footnotetext{
*Correspondence: caojianfangcn@163.com

${ }^{2}$ Department of Computer Science \& Technology, Xinzhou Teachers

University, No. 10 Heping West Street, Xinzhou 034000, China

Full list of author information is available at the end of the article
}

and Northern Dynasties and Tang Dynasty represent an important chapter in the history of world mural paintings [1]. The ancient murals that were discovered and are still preserved in Shanxi, China, represent a total area of more than $25,000 \mathrm{~m}^{2}$. Among existing murals, the largest and most abundant are mural paintings [2]. These murals, which originated in the Han Dynasty and were produced throughout the Southern and Northern Dynasties, were linked to the Tang, Song, Yuan, Ming and Qing dynasties. In addition, the styles were inherited from other dynasties but show variations. Not only do these murals embody the characteristics of paintings in different historical periods, they also play an indispensable role in the study of arts, society, and religion [3]. However, temple murals present varying degrees of flaking deterioration phenomena, damaged as a result of history, climate, 
and other factors. This flaking deterioration not only greatly affects the visual experience of visitors but also represents the beginning of the destruction of cultural heritage, such as murals [4]. The calibration and virtual restoration of deteriorated murals is attracting increased attention from researchers. Researchers can lay the foundation for the virtual display of murals while also providing references for the repair of these murals.

Recently, scholars have achieved various results in addressing this problem facing ancient murals by using intelligent protection. Izzo et al. [5] conducted in-depth research on various materials in Italian fresco painting techniques from different ages, specifically focusing on two main cases, i.e., the murals of Mario Sironi and Edmondo Bacci in Venice, and aimed at understanding the protection needs of these paintings and developing a sustainable protection plan. Sakr et al. [6] studied the influence of and solutions to streptomyces discoloration in mural paintings from ancient Egyptian tombs, thereby laying the foundation for the digital protection of ancient murals. Abdel-Haliem et al. [7] isolated and identified the streptomycetes that mainly affect the discoloration of paintings in ancient Egyptian tombs, thus providing new ideas for the removal of streptomyces. Li et al. [8] investigated the effectiveness of reinforcing mural paintings at the Mogao Grottoes in Dunhuang, China, by conducting on-site inspections to determine if new murals that had been previously repaired presented new forms of deterioration. These studies focused on ancient murals from the perspectives of aesthetics, biology, and chemistry, proposed the need for mural protection and laid the foundation for the protection of ancient murals using intelligent information processing technologies. In research on digital protection processes for ancient murals, Wan [9] studied ancient Chinese Dunhuang murals and designed a pigment color prototype that facilitates the digital conservation of ancient murals. Zhang et al. [10] developed a simulation platform for morphology analysis of ancient mural deterioration. By processing the surfaces of digital murals, Zhang et al. obtained three-dimensional data on murals and proposed a quantitative analysis method to analyze micro changes in the murals. Ren et al. [11] proposed a color clustering and masking algorithm to segment and extract damaged Dunhuang mural regions and designed a mural calibration and repair algorithm for fading and scratches. Tang et al. [12] proposed a method of clustered multi-instance learning to classify mural images from different ages, therein attempting to address the characteristics of Dunhuang mural images, which present strong intraclass variance and background noise. Luo et al. [13] extracted the scale-invariant feature transform (SIFT) and color features of mural images and used support vector machines to automate the classification of mural deterioration in Dunhuang. Li et al. [14] designed a visual analysis tool based on risk management to study single or multiple risks at the scales of sites, caves, walls, and specific risk areas from different perspectives at Mogao Grottoes in Dunhuang for facilitating computer intelligent repair processing. For the automatic calibration of murals, Wang et al. [15] extracted crack information using a top-hat transformation from the images of Tang dynasty mural paintings to realize automatic recognition and calibration. However, this method relied heavily on the selection of structural elements and thus experienced an overcalibration problem. Li et al. [16] proposed an automatic calibration method for mud spot disease in addressing the burial mural erosion problem. However, the texture feature used in this method is only ideal for the calibration of mud spot damage and experiences the problem of insufficient calibration for flaking deterioration. Wu et al. [17] improved the algorithm in [15], increased the scale of the structural elements, and used multiscale calibration of the deterioration; however, the analysis and calculation process of this algorithm is complex, and the time complexity is high. Huang et al. [18] proposed a multipath convolutional neural network (CNN) algorithm to detect erosion damage areas in 89 groups of ancient murals. This study reports the latest research results for the intelligent processing of ancient murals using machine learning methods. Scholars studying the intelligent processing of ancient murals are still in the beginning and exploration stages of this field.

The basic idea of the region growing algorithm [19] is based on predefined criteria for aggregating pixels or subregions into larger regions. The method first starts with "a set of" seeds and subsequently attaches adjacent pixels of a similar nature to the seeds (grayscale or a specific range of color) of the growing area; this method has a wide range of applicability in automatic image segmentation and calibration, especially in the medical field. Ogiela et al. [20] used a region growing algorithm for the automatic segmentation and calibration of the carotid artery bifurcation region. Rajkumar et al. [21] proposed an automated mammogram segmentation based on seed point recognition and an improved region growing algorithm. Cheng et al. [22] used image reconstruction to design a new region growing algorithm based on selfgrowth vector estimation regions. For other areas of an image, the region growing algorithm has various applications. For satellite remote sensing images, Liu et al. [23] used an expanded maxima transformation to improve the region growing algorithm and effectively detected the convective elements of geostationary satellite remote sensing images. To solve the problem of color aliasing and mixed colors in the contour color layer of a scanned topographic map, Liu et al. [24] proposed a contour-line 
color layer separation algorithm based on a fuzzy clustering and region growing algorithm. To address the issue of the optical similarity of adjacent grains or optical aggregates in the precise imaging of minerals in weathered sandstones, which makes grain boundaries difficult to define, Asmussen et al. [25] proposed a seeded region growing algorithm that analyzes 39 transmitted light images of 13 weathered sandstone samples from the Buntsandstein Formation in northwestern Germany. In summary, the region growing algorithm has been successfully applied in image segmentation and calibration, but the application of this algorithm in the segmentation of ancient murals has not been reported. As a special type of image, murals in the contours of an exfoliated area are clearly segmented from other normal areas. Applications using region growing algorithms to demarcate the exfoliation of ancient murals are highly applicable.

The team of authors in this study analyzed ancient temple murals and found that deterioration caused by flaking processes is the most serious type of damage, as the flaking area is relatively large. Compared with other types of damage, the impact of flaking deterioration on the content of the murals is high. In general, the overall structure of a mural is as follows: the lowermost layer is the support (wall), the center layer is the earth layer (normally made from the mixture of sand, cotton, hemp fibers, wheat straw segments, glue, alum, and so on; the surface of which is an approximately $1-\mathrm{mm}$ thick layer of plaster), and the uppermost layer is the painting, as shown in Fig. 1. According to the degree of flaking experienced by the mural, mural flaking can be divided into paint layer flaking (shallow layer flaking, i.e., the part of the earth layer exposed by the mural) and earth layer flaking (deep layer flaking, i.e., the part of the support layer barely exposed by the mural). As shown in Fig. 2, the area marked with a box in the figure is a flaking area. Ancient temple murals combined painting with sculpting and a variety of compositional forms in the creative process, thereby integrating sculptures and paintings. The murals are colorful and have rich variation and profound meaning, and the borders of various regions of the mural are obvious and easily separated, thereby providing new opportunities for the use of computer intelligence processing technology for automatically calibrating the flaking-damaged areas in murals. To protect the abovementioned ancient temple murals, this paper proposes the region growing algorithm fused with threshold segmentation (TS-RG), which automatically calibrates the flaking area in temple murals based on the mural of Kaihua Temple from the Song Dynasty. Based on region growing and threshold segmentation algorithms for calculating each feature mask separately, each feature mask is merged to improve the calibration accuracy. Then, the

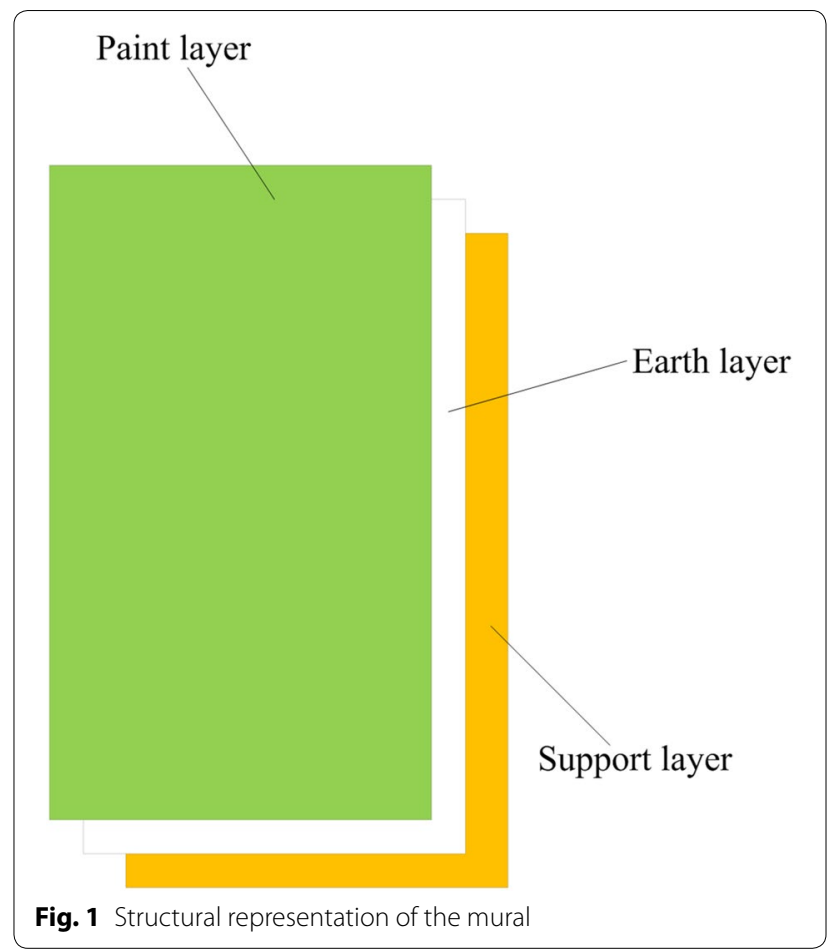

final mask of the flaking-damaged region is obtained via automatic calibration. The experimental results show that the algorithm is more accurate than current calibration algorithms and that user satisfaction is improved.

\section{Methods}

Kaihua Temple is located in Mengshan, $20 \mathrm{~km}$ southwest of Taiyuan in Shanxi, China. This temple was built in the post-Tongzhuang period of the Five Dynasties and rebuilt in the Northern Song Dynasty. Kaihua is a famous ancient temple in Shanxi and one of its most valuable buildings. The Main Shrine Hall is the primary building of the temple. The murals in the temple comprise a total area of $88.2 \mathrm{~m}^{2}$ and are among the largest and most unique murals still existing from the Chinese Song Dynasty [3]. However, due to climate change, natural disasters, and environmental pollution, these murals are facing varying degrees of damage, including smoke damage, fissures, mildew, color fading, and wall detachment. Therefore, the different regions that constitute these mural images have varying degrees of defects, with some on the verge of losing their original appearance and urgently needing to be repaired and protected. No obvious shapes or textures characterize the flaking areas of the temple murals, but the color features are particularly obvious, and the flaking areas are relatively monochromatic. The paint layer flaking areas have a higher brightness and gray value; the earth layer flaking areas have a 


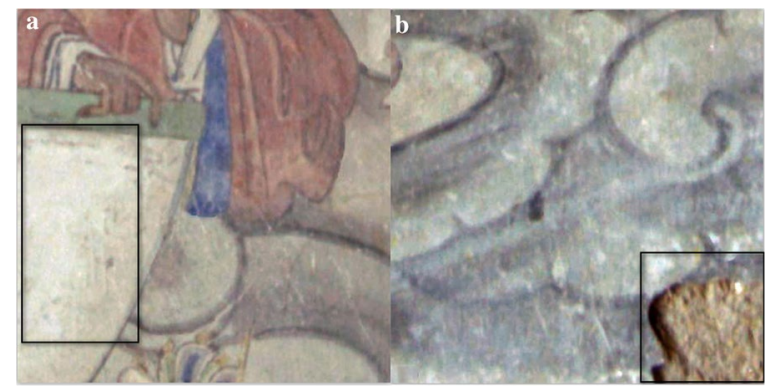

Fig. 2 Example of flaking-related deterioration in an ancient temple mural. a Paint layer flaking. b Earth layer flaking

high saturation and present more obvious color differences. In this paper, temple murals are calibrated using different methods based on their characteristics, i.e., different degrees of flaking deterioration.

\section{Automatic identification and calibration of flaking deterioration in the paint layer}

The paint layer of a temple mural is both based on the earth layer and above the earth layer. The earth layer is a mural surface painted with a plaster with a thickness of approximately $1 \mathrm{~mm}$ that has a color similar to white, with a high brightness and gray value. The identification and calibration of damages to the paint layer are relatively easy. This paper uses a threshold segmentation method to identify such damage. This method transforms the image from the Red-Green-Blue (RGB) color space into the $\mathrm{YCbCr}$ (where $\mathrm{Y}$ refers to the luminance component, $\mathrm{Cb}$ refers to the blue chroma component, and $\mathrm{Cr}$ refers to the red chroma component) color space [7]. Next, grayscale histogram arithmetic is used to obtain the range of brightness values of the intact mural image and the pure earth layer image to determine the threshold range of the flaking area in the paint layer. Then, the method uses Eq. (1) to perform threshold segmentation to calculate the mask of the flaking area. Finally, the mask and the image to be calibrated are added, thus recognizing and calibrating the flaking deterioration in the paint layer.

$$
M_{\text {mask }}= \begin{cases}255, & \tau_{1} \leq S_{i} \leq \tau_{t} \\ 0, & \text { other }\end{cases}
$$

Here, $M_{\text {mask }}$ is the mask of the flaking area, $S_{i}$ is the $Y$ component pixel value of the image to be calibrated in $\mathrm{YCbCr}$ space, and $\tau_{1}$ and $\tau_{2}$ are the lower and upper thresholds in the flaking area, respectively. The extraction results of the grayscale histogram arithmetic are shown in Fig. 3.

Because the brightness of the images obtained differs with different angles, times and illumination intensities, the experimental results have a great influence. Therefore, mural images obtained by frontal shooting under indirect sunlight during the same time period are selected for the experiment. Repeated experiments indicated that the brightness values of the pure earth layer images are distributed between 187 and 221, while the brightness values of the paint layers of intact mural images are mainly distributed between 78 and 174. Therefore, the brightness value range of 187-221 is used as the brightness segmentation threshold; the pixels with a brightness in this range are considered the flaking region, whereas the pixels with a brightness not in this range are not in this region.

\section{Automatic identification and calibration of flaking deterioration in the earth layer $T S$-RG algorithm}

Region growing is the process of combining pixels or subregions into a larger area according to predefined rules; region growing algorithms are very sensitive to the seed point [26]. To improve the accuracy of the mask calculation, the TS-RG algorithm separates the RGB channels of the color mural image. Then, the algorithm uses a $3 \times 3$ window to apply the median-filter method; then, the method applies threshold segmentation to each channel to determine the seed points and utilizes multiple subpoint area growing processes. The eight neighboring points for which the feature similarity satisfies the given conditions are merged until all the pixel points have been processed. Finally, the growth results of each channel region are merged. The TS-RG algorithm process is shown in Fig. 4.

The steps of the algorithm are as follows:

Input: color image to be calibrated $I$, RGB grayscale range of each channel $\varepsilon$, and RGB growth threshold value $T$ of each channel $\varepsilon$.

Output: image color mask to be calibrated $M_{\text {mask }}$.

Step 1. Separate the RGB channels of the image to be calibrated.

Step 2. Perform $3 \times 3$ median filtering and merging for each channel; then, obtain the median filtered image Iffilter.

Step 3. Threshold segmentation determine the grayscale change range in RGB space for each channel $\varepsilon$ and calibrate the gray value of each pixel. The pixel gray value in the $\varepsilon$ range is set to 255 , and the other values are set to 0 , thereby obtaining the threshold segmented image I_out.

Step 4. Determine the seed point $\left(x_{i}, y_{i}\right)$ : a pixel with a gray value of 255 is considered the seed point 

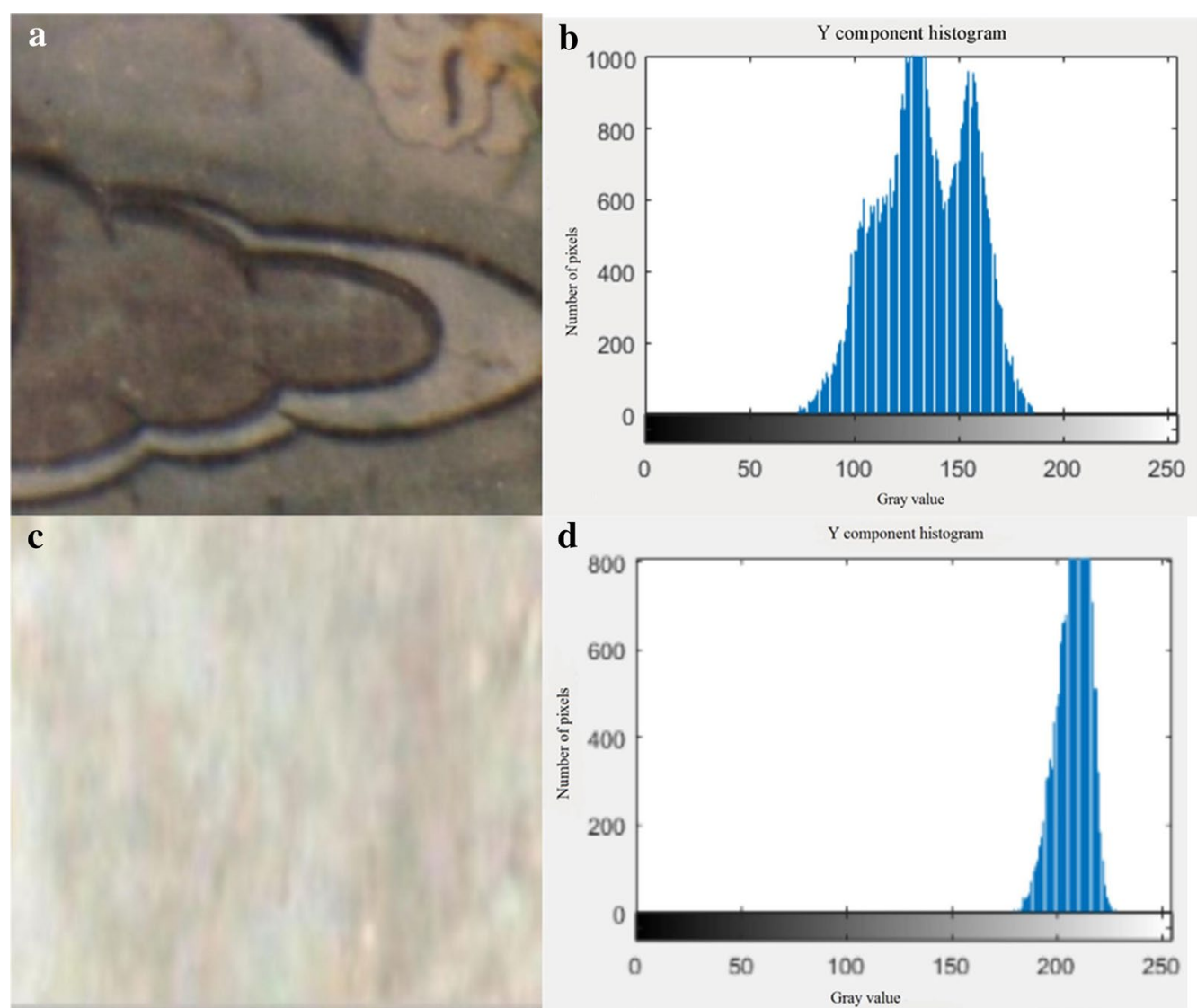

Fig. 3 Earth layer and paint layer luminance histogram. a Paint layer image. b Paint layer luminance histogram. c Earth layer image. $\mathbf{d}$ Earth layer luminance histogram

for the region growing algorithm.

Step 5. I filter, $\left(x_{i}, y_{i}\right)$, and $T$ are used as input parameters for region growing: calculate the gray value of the eight neighboring pixels $\left(x_{i}, y_{i}\right)$ of each seed point and then calculate the averaged value of the pixel gray value in the expanded area. Finally, obtain the minimum value of each seed point min.

Step 6. For each seed point $\left(x_{i}, y_{i}\right)$, if $\min <\mathrm{T}$, the pixel is added to the expanded pixel queue, and the pixel value is set to 255 ; otherwise, the region growing stops, and the mask is obtained.

Step 7. Repeat (5)-(6), individually growing three regions of $R, G$, and $B$ to obtain the mask for each channel.

Step 8. Each channel mask is merged to obtain the color mask of the image to be calibrated $M_{\text {mask }}$

\section{Automatic identification and calibration of earth layer flaking deterioration based on the TS-RG algorithm}

After calibrating for damage to the paint layer of a mural, the most critical task is to identify and calibrate more complex earth layer deterioration. It is impossible to accurately calibrate the threshold segmentation based on the brightness characteristics used in the calibration of the mural paint layer previously described. Therefore, it is necessary to comprehensively analyze the chroma, saturation, color, and other characteristics of the flaking region and then use the proposed TS-RG algorithm to achieve calibration. The process underlying the automatic calibration method for mural earth layer diseases based on the TS-RG algorithm is shown in Fig. 5.

(1) Color mask For the earth layer flaking issue, the exposed part of the mural is the supporting component. The support for temple murals in the Song Dynasty was mostly walls, and the surface was mainly mud, resulting in 

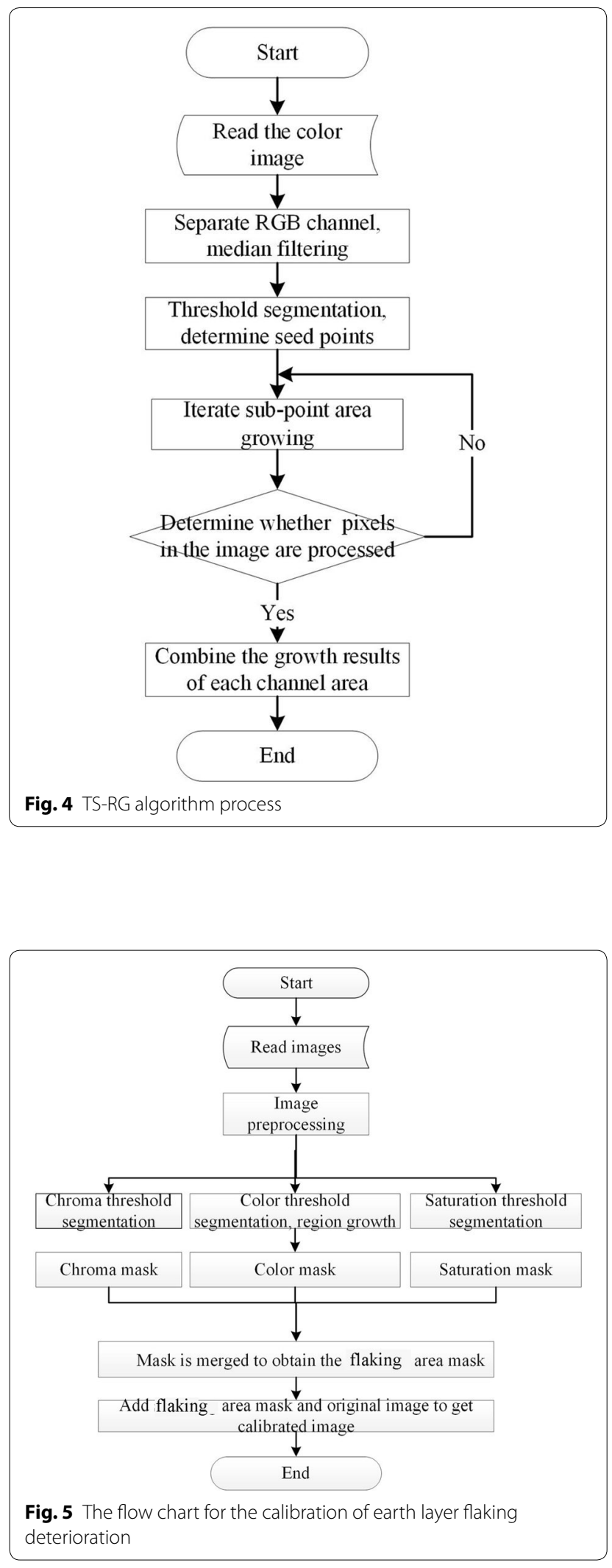

Fig. 5 The flow chart for the calibration of earth layer flaking deterioration the majority of the color in the flaking area to appear light yellow. Therefore, the color range of the flaking area can be set as follows:

$$
\left\{\begin{array}{l}
183 \leq I_{r} \leq 200 \\
159 \leq I_{g} \leq 176 \\
128 \leq I_{b} \leq 146
\end{array}\right.
$$

where $I_{r}, I_{g}$, and $I_{b}$ represent the red, green, and blue components, respectively.

The TS-RG algorithm is used to extract the color mask from the flaking area of the earth layer in the mural as follows:

Step 1. Use a $3 \times 3$ window to perform median filtering on the three channels, i.e., R, G, and B.

Step 2. Traverse the image to find the point that satisfies Eq. (2) and calculate the position of the point in the image $\left(x_{i}, y_{i}\right)$.

Step 3 According to Eq. (3), calculate the regional growth and stopping criterion for the flaking area of the earth layer in the mural.

$\left|f_{k}(m, n)-M\right|_{\min } \leq \tau, \quad k=1,2, \ldots, 8$

In Eq. (3), $M=\frac{\sum_{i=1}^{N} f_{i}(m, n)}{N}$ ( $\mathrm{N}$ is the number of expanded pixel points) represents the average pixel value of the expanded area, $f_{k}(m, n)$ represents the values in the 8 directions near the pixel, and $\tau$ is the threshold value of the regional growth.

The histogram analysis of multiple images with flaking regions found that the variation range of the peak in the $R$ channel is $0-0.1132$. Thus, $\tau_{r}$ takes on the value of 0.1132; simultaneously, $\tau_{g}$ and $\tau_{b}$ take on values of 0.0938 and 0.0899 , respectively.

Step 4 Use the point obtained in Step 2 as a growing point and $\tau_{r}, \tau_{g}$, and $\tau_{b}$ as the thresholds for region growing in each channel to obtain the masks $R_{\text {mask }}, G_{\text {mask }}$, and $B_{\text {mask }}$ per channel. Using Eq. (4), the mask of each channel is calculated, and the final color mask $H_{\text {mask }}$ is obtained

$$
H_{\text {mask }}=R_{\text {mask }} \oplus G_{\text {mask }} \oplus B_{\text {mask }}
$$

(2) Chroma mask The color mask alone is not sufficient to accurately calibrate the flaking area of the earth layer. Due to impurities other than mud in these supporting components, it is impossible to accurately calibrate these 
damaged areas using only color masks. Therefore, for murals where color image features can be fully utilized, the color, saturation and other features are introduced to calibrate these damaged areas. The $\mathrm{YCrCb}$ color space has the characteristics of discreteness, separates brightness and chromaticity and is more suitable for color analysis than RGB space. Therefore, when obtaining the chroma mask of the mural image, this paper first converts the color space of the mural from the RGB space to the $\mathrm{YCrCb}$ color space according to Eq. (5).

$$
\left[\begin{array}{c}
Y \\
C_{b} \\
C_{r}
\end{array}\right]=\left[\begin{array}{ccc}
65.481 & 128.553 & 24.966 \\
-39.797 & -74.203 & 112 \\
112 & -93.786 & -18.214
\end{array}\right] \times\left[\begin{array}{l}
R \\
G \\
G
\end{array}\right]+\left[\begin{array}{c}
16 \\
128 \\
128
\end{array}\right]
$$

Then, the flaking area is identified and calibrated because the range of the chroma distribution $C_{b}, C_{r}$ differs according to the flaking area and perfect area. Similarly, a group of images that only contain an earth layer flaking area and intact images are analyzed based on their chromaticity histograms and threshold values, as shown in Fig. 6.

Several experiments show that when the values of $C_{r}$ and $C_{b}$ are 142 and 118, respectively, the flaking and intact areas can be separated. Thus, these values are used as thresholds for the chrominance mask $\tau_{c r}$ and $\tau_{c b}$. Assume that the image to be calibrated is $f_{b i}$. The chromaticity is denoted by $C_{b i}$ and $C_{r i}$. Then, $f_{b i}$ can be used to obtain the chroma mask $C_{\text {mask }}$ :

$$
C_{\text {mask }}= \begin{cases}255, & \tau_{c b} \leq C_{b i} \wedge \tau_{c r} \leq_{\mathrm{ri}} \\ 0, & \text { other }\end{cases}
$$

(3) Saturation mask The hue, saturation, and value (HSV) space can intuitively express the shades and vividness of colors and facilitate comparisons between colors [27]. When obtaining the saturation mask, this work first converts the image to be calibrated from the RGB space to the HSV space and then uses the same method as that used for the chroma mask to determine a saturation threshold of the image of 0.2012. This method can separate the flaking area from the intact area. According to Eq. (7), this method calculates the saturation mask $S_{\text {mask }}$ of the mural image:

$$
S_{\text {mask }}= \begin{cases}255, & S_{i}<\tau_{s} \\ 0, & S_{i}>\tau_{\mathrm{s}}\end{cases}
$$

(4) Mask fusion and flaking area calibration Since only the chroma or saturation is used to obtain the mask of the flaking area, there will be an excessive number of calibration areas. The intact areas with similar chroma, saturation, and threshold values are also calibrated. However, the color features alone cannot be used to calibrate areas where the grayscale of the flaking area changes
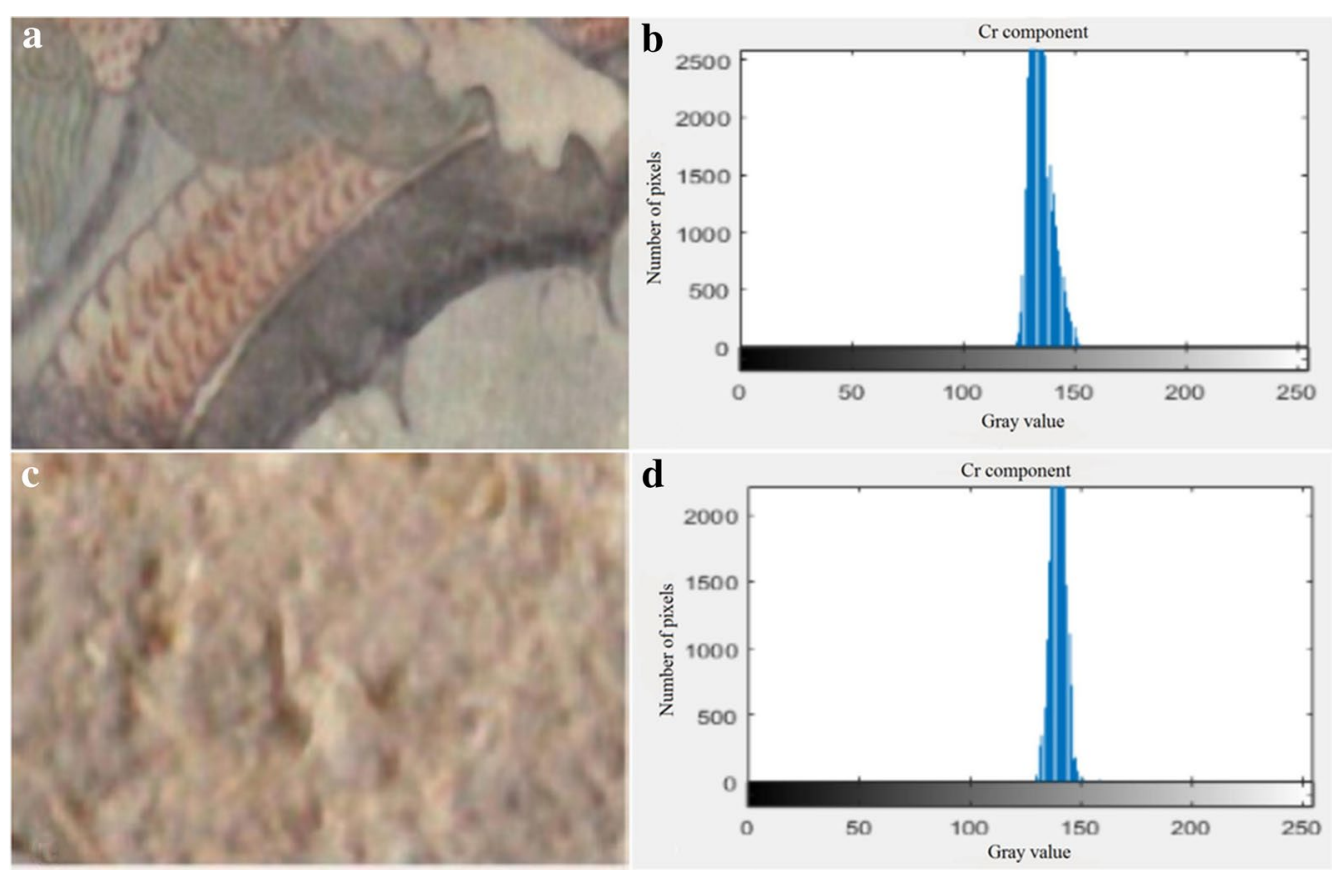

Fig. 6 Contrast diagram of the Hue histogram. a Intact mural image. $\mathbf{b}$ Hue histogram of intact mural. c Earth layer flaking area image. $\mathbf{d}$ Hue histogram of earth layer flaking area 
significantly. Therefore, by considering the information of at least two colors, chrominance, and saturation masks, the calibration accuracy improves. In this paper, according to Eq. (8), the extracted color, chrominance, and saturation masks $H_{\text {mask }}, C_{\text {mask }}$, and $S_{\text {mask }}$ are calculated to obtain the mask of the final flaking area $M_{\text {mask }}$.

$$
M_{\text {mask }}=H_{\text {mask }} \otimes C_{\text {mask }} \oplus H_{\text {mask }} \otimes S_{\text {mask }} \oplus C_{\text {mask }} \otimes S_{\text {mask }}
$$

Then, the mask is added to the original image according to Eq. 9 to automatically calibrate the flaking area.

$$
f=f_{b} \oplus M_{\text {mask }}
$$

\section{Experimental design}

This article takes the temple murals from the Song Dynasty in Kaihua Temple as the research object. The simulation experiment is based on the Matlab2016 platform on a Windows system with the following hardware environment: Intel(R) Core(TM) i5-4570 CPU @ 3.20 $\mathrm{GHz}$ processor, $4 \mathrm{~GB}$ memory and $500 \mathrm{G}$ hard disk. Aiming to investigate two different degrees of flaking deterioration and the methods in references [15] and [16], these experiments were compared from several perspectives, including calibration effects, calibration errors, and noise calibration errors. The experiment was conducted on a mural image database consisting of 100 images with a resolution of $2600 \times 2600$ (30 pieces of murals with flaking deterioration in the paint layer and 70 pieces of murals with flaking deterioration in the earth layer). Before the experiment, all 100 experimental images were manually calibrated with respect to the damaged area. Each image was calibrated by several experts from the Wudang Mountain Ancient Culture Research Centre, who were not aware of the purpose of the current study. When variations occurred, the final calibration outcomes were determined according to the principle of the minority subordinate to the majority.

To accurately evaluate the proposed algorithm performance, this paper first verifies the calibration effect. For relatively complex earth layer deterioration calibrations, this paper also uses the leave-one-out strategy [28] to separate sample images and test images, i.e., a randomly selected mural image is used as the test image, and the remaining 69 images form the sample set. The calibration area of the test image is calculated by the autocalibration algorithm of the earth layer flaking deterioration based on the TS-RG algorithm. The algorithm can ultimately be used to obtain the earth layer flaking deterioration area of all 70 mural images. We calculated the Euclidean distance between the center of the flaking deterioration area calibrated by this method and the center of the flaking deterioration area calibrated manually beforehand as a criterion for the performance evaluation of the algorithm.

\section{Results and discussion}

\section{Automatic paint layer flaking calibration experiment}

The flaking area is more obvious for paint layer flaking deterioration than for earth layer flaking deterioration. This paper selects images of paint layer flaking deterioration affecting the temple murals from the Song Dynasty in Kaihua Temple and conducts an experiment

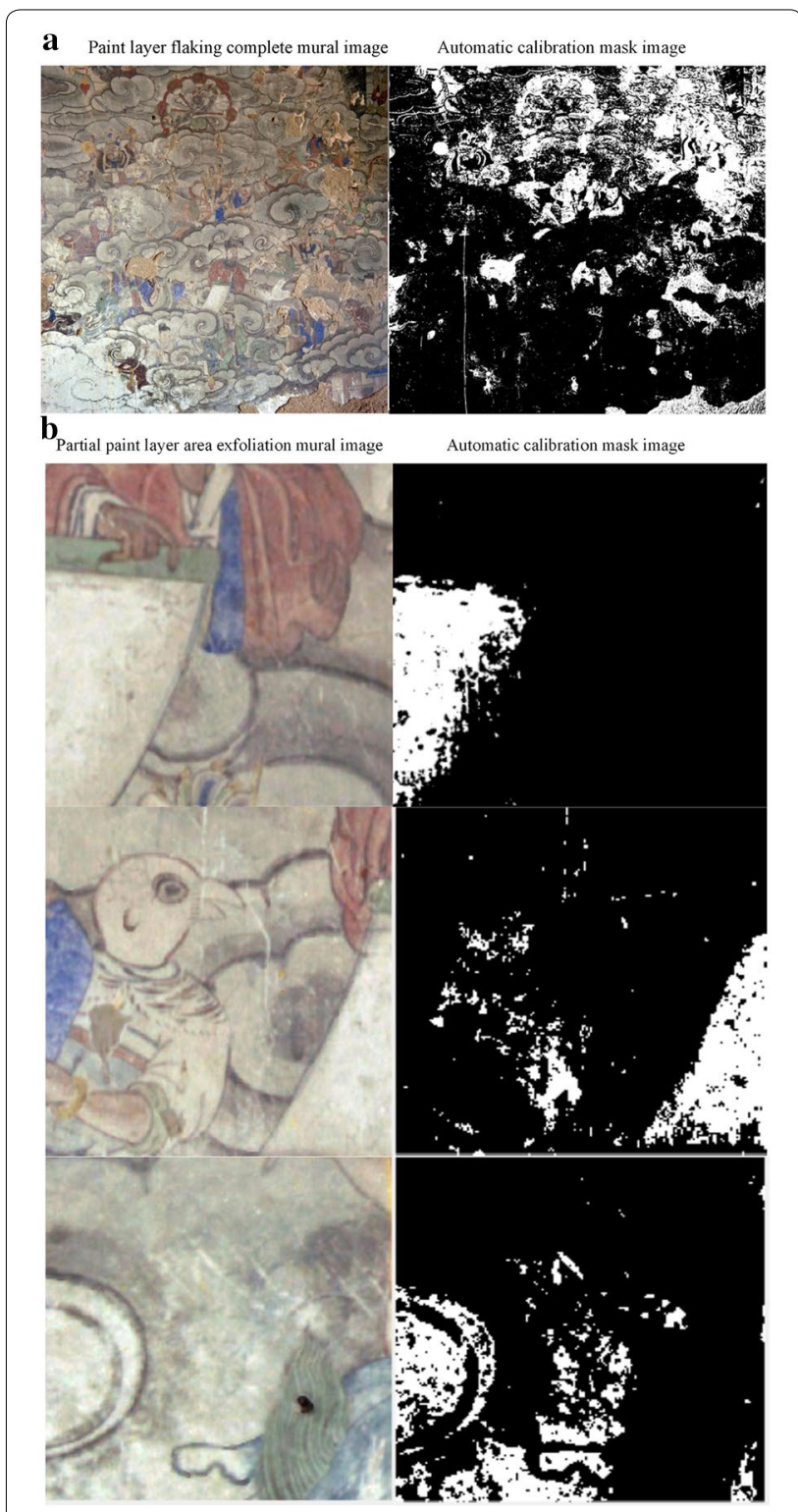

Fig. 7 Example of automatic recognition and calibration of flaking-related deterioration in a paint layer. a Whole width of mural. b Local mural area 
based on the threshold segmentation method. The results are shown in Fig. 7.

From the calibration results in Fig. 7, it can be seen that when the pre-prepared segmentation method is used to calibrate the flaking deterioration in the paint layer, even if there are very small faded areas in the image to be calibrated, the overall calibration result is satisfactory, and incorrectly calibrated areas do not appear, thereby achieving the desired effect.

\section{Automatic earth layer flaking calibration experiment}

The TS-RG algorithm proposed in this paper was used to automatically calibrate the mural images of earth layer flaking deterioration for murals in the Kaihua Temple from the Song Dynasty. The experimental results are shown in Fig. 8.

The automatic calibration results of the TS-RG algorithm were compared with the experts' manual calibration. Based on the different degrees of consistency, user satisfaction was quantitatively divided into five levels: very dissatisfied $(<50 \%)$, dissatisfied $(50-70 \%)$, basically satisfied (70-85\%), satisfied (85-95\%), and very satisfied $(\geq 95 \%)$.

Figure 8 shows that, for the calibration of earth layer flaking deterioration on the mural, only the mask generated by saturation and chromaticity exhibits too much calibration, and the user satisfaction is basically satisfactory. Only the masks generated by the color features calibrate the defects with insufficient calibration, and the user satisfaction level for these masks is not satisfactory. Therefore, the use of only a single feature results in a large calibration error, which cannot meet the calibration accuracy and user satisfaction requirements. In this paper, the three masks are fused so that the calibrated flaking area meets at least two requirements of color, saturation and chromaticity. Currently, these flaking area calibration results are more accurate than the singlecharacteristic calibration results, and the user satisfaction

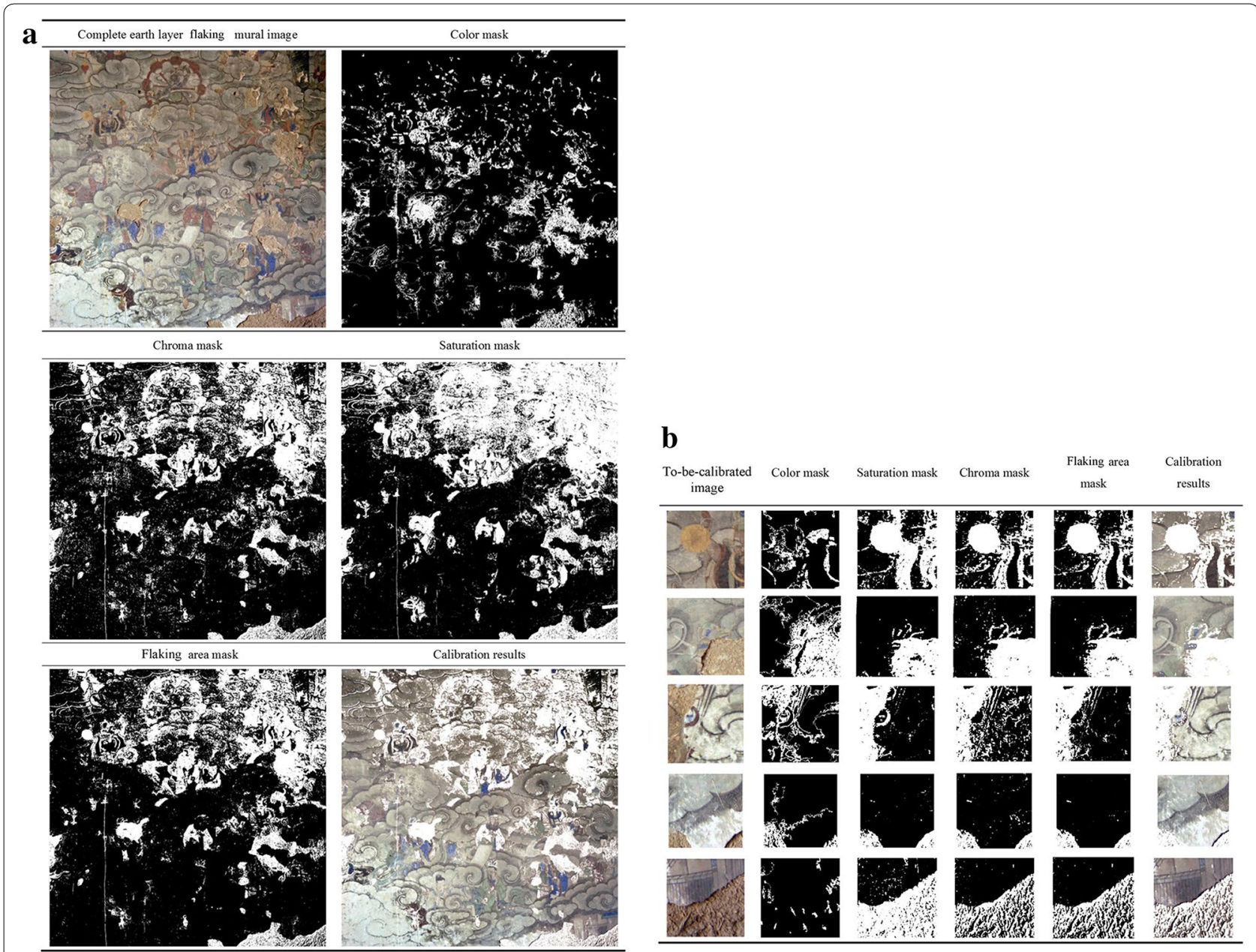

Fig. 8 Automatic identification and calibration of flaking-related earth layer deterioration. a Whole width of mural. b Local mural area 

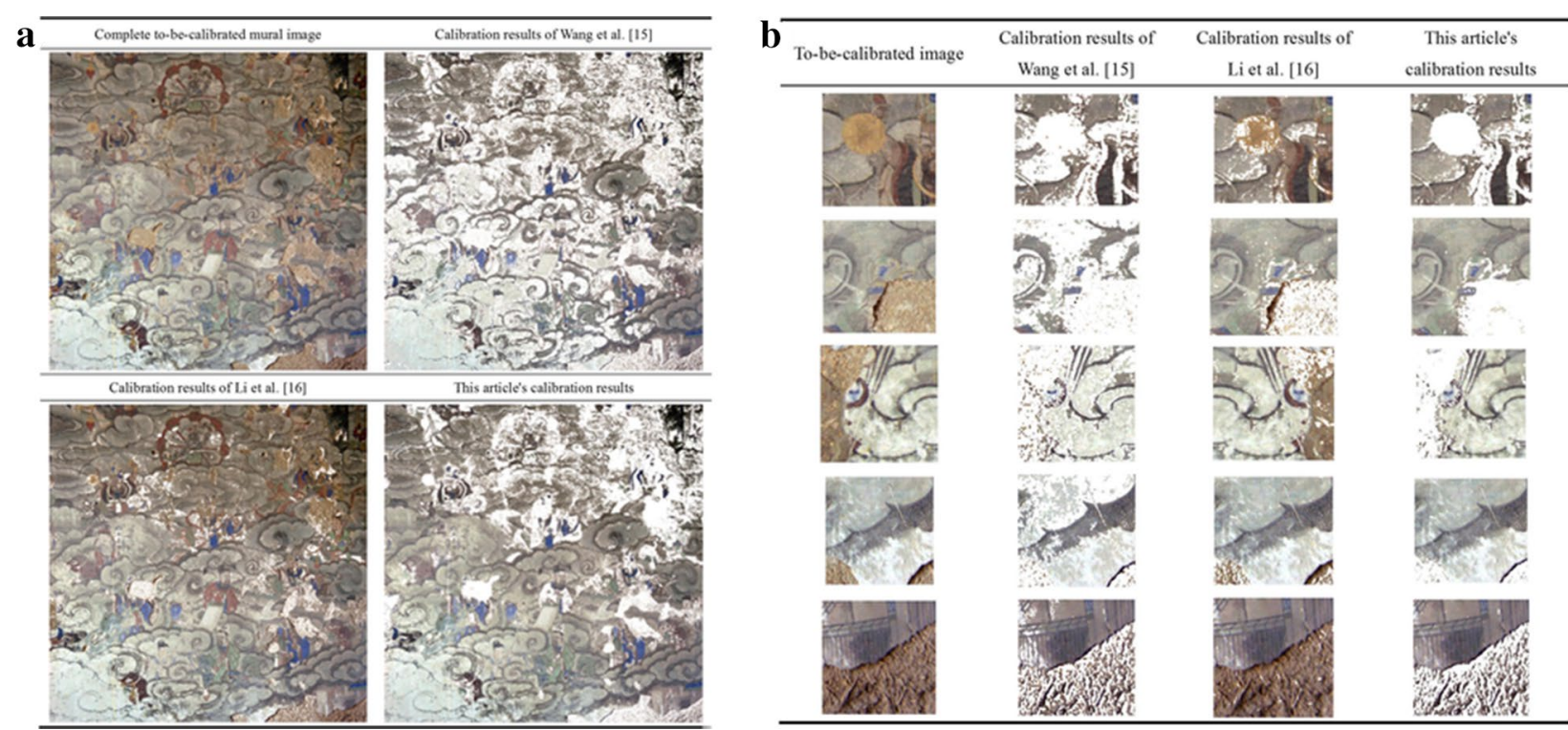

Fig. 9 Comparison of different calibration methods. a Whole width of mural. b Local mural area

\begin{tabular}{|c|c|c|}
\hline Method & $\begin{array}{l}\text { Mean error } \\
\text { (pixel) }\end{array}$ & $\begin{array}{l}\text { Error } \\
\text { standard } \\
\text { variance }\end{array}$ \\
\hline The method by Wang et al. [15] & 2.69 & 3.97 \\
\hline The method by Li [16] & 2.46 & 2.71 \\
\hline The method in this article & 1.91 & 1.82 \\
\hline
\end{tabular}

level is very satisfactory. This method was applied to the experimental image in Fig. 7. In addition, to further verify the effectiveness of the method proposed in this paper, the results with respect to the deterioration of murals from the Song Dynasty in Kaihua Temple were compared with those of the methods of Wang et al. [15] and Li et al. [16], which are commonly used in mural damage calibration. The results are shown in Fig. 9.

The comparison of the calibration results in Fig. 9 clearly shows that, in the method proposed by Wang et al. [15], a nonflaking region appears after the threshold segmentation; then, the connected component labeling causes the area outside of the flaking region to be calibrated. There are excessive calibration problems. The calibration method of Li et al. [16] only uses the texture and brightness features for threshold segmentation, and the partially flaking area was not calibrated, resulting in the presence of missing areas in the flaking deterioration calibration. With the TS-RG algorithm proposed in this paper, we first used the color features for the threshold segmentation to determine the flaking seed point; then, we performed region growing to determine the color mask and subsequently merged this mask with the saturation and chromaticity masks determined by threshold segmentation, thus increasing the calibration result accuracy.

In addition, for comparison with the methods of Wang et al. [15] and Li et al. [16], the autocalibration error tests for 70 samples containing earth layer flaking deteriorations are shown in Table 1.

The data in Table 1 demonstrate that the method used in this paper achieves the smallest average error and error standard deviation. Although the average errors of the three methods are not very large, the error standard deviations of the methods by Wang et al. [15] and Li et al. [16] were 3.97 and 2.71, respectively, far higher than the error standard deviation achieved by the method proposed in this paper. This result demonstrates that the algorithm in this paper can be effectively used to calibrate differences between the pixel point errors and average area values. The algorithm is relatively stable and effective at automatically calibrating the earth layer flaking deterioration of murals.

To better verify the robustness of the proposed algorithm, an experiment was performed to compare $10 \%$ salt and pepper noise and Gaussian noise with a mean square error of 10 [29]. The results are shown in Table 2.

The data in Table 2 show that, although the average errors and error standard deviations of the various methods decrease after a certain amount of noise is added, the method proposed in this paper presents the smallest 
Table 2 Comparison of performances of different algorithms after adding noise

\begin{tabular}{|c|c|c|c|}
\hline Method & Without noise & $10 \%$ salt and pepper noise & $\begin{array}{l}\text { Gaussian noise } \\
\text { with an average square } \\
\text { deviation of } 10\end{array}$ \\
\hline \multicolumn{4}{|l|}{ Method in reference [15] } \\
\hline Mean error (pixels) & 2.69 & 3.04 & 2.98 \\
\hline Error standard variance & 3.97 & 4.15 & 4.09 \\
\hline \multicolumn{4}{|l|}{ Method in reference [16] } \\
\hline Mean error (pixels) & 2.46 & 2.83 & 2.70 \\
\hline Error standard variance & 2.71 & 3.11 & 2.99 \\
\hline \multicolumn{4}{|l|}{ Method of this article } \\
\hline Mean error (pixels) & 1.91 & 1.97 & 1.96 \\
\hline Error standard variance & 1.82 & 1.85 & 1.85 \\
\hline
\end{tabular}

decrease, and the error standard deviation changes the least. This result further demonstrates the robustness of the calibration method proposed in this paper.

\section{Conclusion}

This paper focuses on the flaking problem of mural paintings in Kaihua Temple from the Song Dynasty. Through the in-depth analysis and study of the color, saturation, chroma, brightness and other features of the flaking area, this paper proposes an automatic calibration method based on the TS-RG algorithm for flaking images in murals. In the MATLAB platform, the automatic identification and calibration of flaking deterioration in ancient temple murals were realized, thereby laying the foundation for the virtual restoration of murals and playing a supporting role in promoting the digital protection of ancient cultural heritage. However, in flaking deteriorations affecting murals, part of the flaking area may contain overlapping flaking in the paint layer and the earth layer. In this type of overlapping region, the range of variations of the chroma and brightness of the flaking area is relatively large, and current thresholds cannot accurately calibrate both characteristics. Therefore, the next step in this research is to further optimize the threshold to improve the calibration accuracy and provide a more accurate repair range for the repair process. This would make the repaired murals look more natural and closer to the true intentions of the mural creators. Moreover, because the mural images selected in this paper were taken under the same illumination, time period and angle conditions, the image shadow and blur problems caused by variations in these factors are not considered. Therefore, the next step will be to consider these issues during the image preprocessing phase and reduce the limitations of the algorithm.

\section{Abbreviations}

RGB: red-green-blue; HSV: hue, saturation, and value.

\section{Authors' contributions}

All authors have made contributions to the current work. CJF devised the study plan and led the writing of the article, LYF and CHY conducted the experiment and collected the data. ZQ conducted the analysis, and CJF supervised the whole process and gave constructive advice. All authors read and approved the final manuscript.

\section{Author details}

1 School of Computer Science \& Technology, Taiyuan University of Science and Technology, Taiyuan 030024, China. ${ }^{2}$ Department of Computer Science \& Technology, Xinzhou Teachers University, No. 10 Heping West Street, Xinzhou 034000, China.

\section{Acknowledgements}

The authors thank the colleagues who assisted during the work.

\section{Competing interests}

The authors declare that they have no competing interests.

\section{Availability of data and materials}

The datasets used and/or analyzed in the current study are available from the corresponding author by reasonable request.

\section{Funding}

This study was funded by the Natural Science Foundation of Shanxi Province (201701D21059), the Art Disciplinary Project of Shanxi Province (2017F06) and the 13th Five-Year Education Science Project of Shanxi Province (GH-17059).

\section{Publisher's Note}

Springer Nature remains neutral with regard to jurisdictional claims in published maps and institutional affiliations.

Received: 6 August 2018 Accepted: 21 November 2018

Published online: 27 November 2018

\section{References}

1. Nicola M, Aceto M, Gheroldi V, Gobetto R, Chiari G. Egyptian blue in the Castelseprio mural painting cycle. Imaging and evidence of a non-traditional manufacture. J Archaeol Sci Rep. 2018;19:465-75.

2. Zhang HJ, Wang JY, Shanxi Li SF. Mural and cultural relics protection-an interview with Mr. Chai Zejun, an ancient expert. Arch Shanxi. 2012;1:816 (in Chinese).

3. Zhang Z. Informal discussion on Shanxi Murals. Arch Shanxi. 2013;1:12-20 (in Chinese) 
4. Yang XH. An analysis of line-drawing characteristics of the Shuilu Temple Murals in Shanxi. Arch Shanxi. 2016;3:14-6 (in Chinese).

5. Izzo FC, Falchi L, Zendri E, Biscontin G. A study on materials and painting techniques of 1930s Italian mural paintings: two cases by Mario Sironi and Edmondo Bacci in Venice. England: Cambridge Scholar Publishing; 2015. p. 35-51.

6. Sakr AA, Ali MF, Ghaly MF. Discoloration of ancient Egyptian mural paintings by streptomyces strains and methods of its removal. Int I Conserv Sci. 2012:3:249-58.

7. Abdel-Haliem MEF, Sakr AA, Ali MF, Ghaly MF, Sohlenkamp C. Characterization of Streptomyces isolates causing colour changes of mural paintings in ancient Egyptian tombs. Microbiol Res. 2013;168:428-37.

8. Li J, Zhang H, Fan Z, He X, He S, Sun M, et al. Investigation of the renewed diseases on murals at Mogao Grottoes. Heritage Science. 2013;1:31-5.

9. Wan X. Prototype of a pigments color chart for the digital conservation of ancient murals. J Electron Imaging. 2017;26:023013.

10. Zhang Y, Wang J, Liu H, Wang X. A non-destructive approach using MatLab software for morphology analysis of ancient mural deterioration. Stud Conserv. 2016;62:1-9.

11. Ren XK, Deng LK. Murals inpainting based on color clustering image segmentation and the improved FMM algorithm. Comput Eng Sci. 2014;36:298-302 (in Chinese with English abstract).

12. Tang DW, Lu DM, Xu DQ, Yang B. Clustered multiple instance learning for mural image classification. J Image Gr. 2014;19:708-15 (in Chinese with English abstract).

13. Luo W, Sun J, Jiang J, Wang C. Auto-classification of mural disease images using bag of word model. J Comput Inf Syst. 2014;10:1305-14.

14. Li H, Zhang J, Sun J. A visual analytics approach for deterioration risk analysis of ancient frescoes. JVis. 2016;19:529-42.

15. Wang $\mathrm{K}$, Wang HQ, Wu M. Virtual restoration method of cracks in mural. Comput Eng Appl. 2014:50:136-9 (in Chinese with English abstract).

16. Li CY. Automatic recognition and virtual restoration of mud spot of the murals. Thesis, Xi' an University of Architecture and Technology, 2015. (in Chinese with English abstract).

17. Wu M, Wang HQ, LiWY. Research on multi-scale detection and image inpainting of Tang dynasty tomb murals. Comput Eng Appl. 2016;52:16974 (in Chinese with English abstract).
18. Huang R, Feng W, Fan MY, Guo Q, Sun JZ. Learning multi-path CNN for mural deterioration detection. J Ambient Intell Hum Comput. 2017:27:1-8.

19. Ma J, Son JB, Hazle JD. An improved region growing algorithm for phase correction in MRI. Magn Reson Med. 2016;72:519-29.

20. Ogiela MR, Hachaj T. Automatic segmentation of the carotid artery bifurcation region with a region-growing approach. J Electron Imaging. 2013;22:3029-36.

21. Rajkumar K, Raju G. Automated mammogram segmentation using seed point identification and modified region growing algorithm. Nat Rev Mol Cell Biolog. 2015;10:423-9.

22. Cheng C, Zou C, Liang C, Liu X, Zheng H. Fat-water separation using a region-growing algorithm with self-feeding phasor estimation. Magn Reson Med. 2017;77:2390-401.

23. Liu J, Ma C, Liu C, Qin D, Gu X. An extended maxima transform-based region growing algorithm for convective cell detection on satellite images. Remote Sens Lett. 2014;5:971-80.

24. Liu T, Miao Q, Xu P, Tong Y, Song J, Xia G, et al. Contour-line color layer separation algorithm based on fuzzy clustering and region growing. Comput Geosci. 2016:88:41-53.

25. Asmussen P, Conrad O, Günther A, Kirsch M, Riller U. Semi-automatic segmentation of petrographic thin section images using a "seeded-region growing algorithm" with an application to characterize wheathered subarkose sandstone. Comput Geosci. 2015;83:89-99.

26. FabijaSka A. Yarn image segmentation using the region growing algorithm. Meas Sci Technol. 2011;22:114024.

27. Chernov V, Alander J, Bochko V. Integer-based accurate conversion between RGB and HSV color spaces. Comput Electr Eng. 2015;46:328-37.

28. Zhang T. Leave-one-out bounds for kernel methods. Neural Comput. 2014;15:1397-437.

29. Menzel C, Haynleichsenring G, Redies C, Kovács G. Noise can be good: visual adaptation to noise with different Fourier power spectrum characteristics affects the electrophysiological correlates of face processing. J Vis. 2015;15:1198.

\section{Submit your manuscript to a SpringerOpen ${ }^{\odot}$ journal and benefit from:}

- Convenient online submission

- Rigorous peer review

- Open access: articles freely available online

- High visibility within the field

- Retaining the copyright to your article

Submit your next manuscript at $\boldsymbol{\nabla}$ springeropen.com 